SECTION 5. Innovative technologies in science.

Korneyev Andrey Mastislavovich candidate of technical Sciences, associate Professor, Lipetsk state technical University, Russia

Abdullakh Lutfi Salekh postgraduate student Lipetsk state technical University, Russia d.48@,rambler.ru Retivykh Sergey Yuryevich student Lipetsk state technical University, Russia

Smetannikova Tatiana Andreevna postgraduate student Lipetsk State Pedagogical University, Russia

\title{
CONTROL APPROPRIATE TOLERANCES MULTIVARIATE QUALITY
}

Abstract: Now developed a number of standards governing the properties of a particular type of finished product. Consequently, all the quality indicators to be monitored at the stage of manufacture. Often, however, we study the quality of the one-dimensional case, there is only one parameter of the products. This article describes how to control a multidimensional quality.

Key words: automation, design, system, generation, the process.

\section{УДК 519.718}

\section{КОНТРОЛЬ СООТВЕТСТВИЯ ДОПУСКУ МНОГОМЕРНОГО КАЧЕСТВА}

Аннотация: Сейчас разработано множество стандартов, регламентирующих свойства того или иного вида готовой продукции. Следовательно, все показатели качества должны отслеживаться еще на этапе изготовления. Однако зачастую изучаются вопросы качества в одномерном случае, то есть только для одного параметра продукции. $B$ данной статье рассмотрены вопросы контроля многомерного качества.

Ключевые слова: автоматизация, дизайн, системьг; поколение; прочесс.

\section{Статистические методы регулирования технологических процессов}

Статистическое регулирование технологических процессов - корректирование значений параметров технологического процесса по результатам выборочного контроля контролируемых параметров, осуществляемое для технологического обеспечения требуемого уровня качества. При этом технологический процесс должен быть статистически управляемым и стабильным [1].

Определение «статическая управляемость» подразумевает, что изменения контролируемых параметров продукции носят случайный, несистематический характер. Во многих случаях принимают, что эти изменения подчиняются нормальному закону распределения, поскольку он наиболее распространен на практике. При влиянии 
большого числа слабо взаимосвязанных факторов распределение центрированного и нормированного результата также стремится к нормальному.

Непрерывная случайная величина $\mathrm{X}$, принимающая значения на вещественной оси от $-\infty$ до $+\infty$, имеет нормальное распределение, если ее плотность распределения описывается уравнением:

$$
\varphi\left(x ; \mu, \sigma^{2}\right)=\frac{1}{\sigma \sqrt{2 \pi}} e^{-\frac{(x-\mu)^{2}}{2 \sigma^{2}}}
$$

где $\mu$ - математическое ожидание случайной величины $\mathrm{X}, \sigma$ - среднее квадратичное отклонение случайной величины X.

Кривая нормального распределения характеризуется двумя параметрами: $\mu$ и $\sigma$. $\mu$ отвечает за смещение графика плотности вдоль оси абсцисс, а $\sigma$ определяет форму кривой - чем больше $\sigma$, тем кривая становится более плоской. В случае $\mu=0$ и $\sigma=1$ нормальное распределение является стандартным.

\section{Контрольные карты}

Техническим вспомогательным средством статистического регулирования являются контрольные карты, позволяющие наглядно отобразить ход производственного процесса на диаграмме и таким образом выявить нарушения технологии. Цель контрольных карт - обнаружить неестественные изменения в данных из повторяющихся процессов и дать критерии для обнаружения отсутствия статистической управляемости [2].

Различают контрольные карты по количественным и качественным признакам в зависимости от того, допускает ли признак только качественную оценку или поддается количественному измерению.

Предположим, что показатель качества X имеет нормальное распределение с математическим ожиданием $\mu$ и средним квадратическим отклонением $\sigma$. Задача определения контрольных границ на контрольной карте средних арифметических значений сводится к нахождению границ критической области при проверке на уровне значимости $\alpha$ нулевой гипотезы $H_{0}: \mu=x_{c p}$ (среднее значение параметра распределения генеральной совокупности равно $\mu$ ) против конкурирующей гипотезы $H_{0}: \mu \neq x_{c p}$.

Периодически для контроля отбирается постоянное число изделий $\mathrm{n}$, по которым определяется среднее арифметическое. Пусть по каждой выборке рассчитаны средние $\overline{x_{i}}$, где $i=1,2 \ldots n$. Параметры технологического процесса $\mu_{0}$ и $\sigma$ могут быть известны из технических условий, иначе они оцениваются в ходе процесса. Оценка $\overline{x_{i}}$ среднего значения уровня настройки $\mu_{0}$, равна:

$$
\overline{\overline{x_{i}}}=\frac{1}{k} \sum_{i=1}^{k} \overline{x_{i}}=\frac{1}{n k} \sum_{i=1}^{k} \sum_{j=1}^{n} x_{i j}
$$

Для оценки параметра $\sigma$ вычисляется несмещенная оценка дисперсии

$$
s_{i}^{2}=\frac{1}{n-1} \sum_{j}\left(x_{i j}-\overline{x_{i}}\right)^{2}
$$

При заданном уровне значимости $\alpha$ для проверки гипотезы используется выборочная характеристика Стьюдента

$$
T=\frac{\bar{x}_{i}-\overline{x_{i}}}{s} \sqrt{n}
$$


Тогда границы критической области равны:

$$
\bar{x} \pm z_{\alpha} \frac{\sigma}{\sqrt{n}}
$$

где $z_{\alpha}$ - определяется по таблице интегральной функции $\Phi(\mathrm{t})$.

Устанавливая таким образом критическую область для гипотезы $H_{0}$, с вероятностью $\alpha$ допускается ошибка 1-го рода - несмотря на истинность гипотезы и отсутствие необходимости подналадки, определяется нарушение технологического процесса. Часто значение для величины $\alpha$ принимают 0,0027 , чему соответствует $z_{0,0027}$ $=3$, тогда интервал критической области составляет $6 \sigma .[3]$

\section{Определение многомерного качества}

Качество изделия обычно характеризуется несколькими параметрами. Использование независимых критериев зачастую может приводить к значительным погрешностям, что обуславливается влиянием параметров друг на друга. Оценивая доверительные области, в данном случае получаем прямоугольный n-мерный параллелепипед, стороны которого определяются границами регулирования контрольных карт. С учетом корреляционных связей доверительная область в действительности при многомерном нормальном распределении показателей представляет собой эллипсоид, главные оси которого повернуты относительно осей параллелепипеда. Также невозможно определение совместного уровня значимости. [2, 3]

Для решения этих проблем применяется контрольная карта Хотеллинга. Для каждой $i-\breve{u}$ выборки рассчитывается статистика

$$
T_{i}^{2}=n\left(\bar{X}_{i}-\mu_{0}\right)^{T} S^{-1}\left(\bar{X}_{i}-\mu_{0}\right),
$$

где $\mathrm{n}$ - объем выборки, $\mathrm{m}$ - количество выборок, $\mathrm{p}$ - количество показателей, $\bar{X}_{i}=\left(\bar{x}_{i 1}, \bar{x}_{i 2}, \ldots, \bar{x}_{i p}\right)$ - вектор средних мгновенных выборок, $\mu_{0}=\left(\mu_{1}, \mu_{2}, \ldots, \mu_{p}\right)^{T}$ вектор целевых средних.

Оценки $S$ компонент ковариационно матрицы $C$ размерности $p \times p$, определяющие рассеивание показателей качества и степень тесноты их связи вычисляются по формуле:

$$
s_{i k}=\frac{1}{m(n-1)} \sum_{t=1}^{m} \sum_{i=1}^{n}\left(x_{i j t}-\mu_{j}\right)\left(x_{i k t}-\mu_{k}\right), \quad j, k=1 \ldots p
$$

При нормальном ходе процесса должно выполняться условие

$$
T_{t}^{2}<T_{\kappa p}^{2}
$$

При неизвестной ковариационной матрице статистика

$$
F=\frac{n-p}{p(n-1)} T^{2}
$$

имеет нецентральное F-распределение Фишера с $p$ и $n-p$ степенями свободы

$$
T_{\kappa p}^{2}=\frac{F(p ; n-p) \cdot p(n-1)}{n-p}
$$

Допустимая область для контрольной карты Хотеллинга оказывается меньше, чем для карты средних значений. Это хорошо видно на двумерном примере. Пусть исходными данными являются показатели производства стали марки DC04 в 3 
выборках по 10 измерений (табл. 1). По предварительным исследованиям номинальные показатели температуры смотки и времени нагрева сляба в печи равны 198,3 и 583 соответственно. Зададим $\alpha=0,05$.

Критическое значение статистики Хоттелинга составляет 10,035, результаты расчетов для выборок представлены в табл.1.

Таблица 1.

Показатели производства стали

\begin{tabular}{|c|c|c|c|c|c|c|c|c|c|c|c|}
\hline & 1 & 2 & 3 & 4 & 5 & 6 & 7 & 8 & 9 & 10 & $T_{t}^{2}$ \\
\hline \multirow{2}{*}{1} & 203,1 & 193,4 & 195,9 & 202,3 & 191,5 & 194,0 & 201,7 & 189,5 & 192,0 & 199,8 & \multirow{2}{*}{2,206} \\
\cline { 2 - 14 } & 584 & 583 & 578 & 582 & 586 & 586 & 584 & 585 & 597 & 584 & \\
\hline \multirow{2}{*}{2} & 207,1 & 198,4 & 205,2 & 201,8 & 191,9 & 206,2 & 197,7 & 196,6 & 195,5 & 205,7 & \multirow{2}{*}{4,989} \\
\cline { 2 - 12 } & 578 & 593 & 576 & 583 & 591 & 596 & 580 & 584 & 589 & 586 & \\
\hline \multirow{2}{*}{3} & 199,5 & 196,4 & 200,3 & 210,6 & 204,9 & 199,6 & 208,8 & 205,3 & 198,4 & 198,8 & \multirow{2}{*}{18,059} \\
\cline { 2 - 11 } & 580 & 591 & 585 & 597 & 606 & 601 & 620 & 617 & 611 & 597 & \\
\hline
\end{tabular}

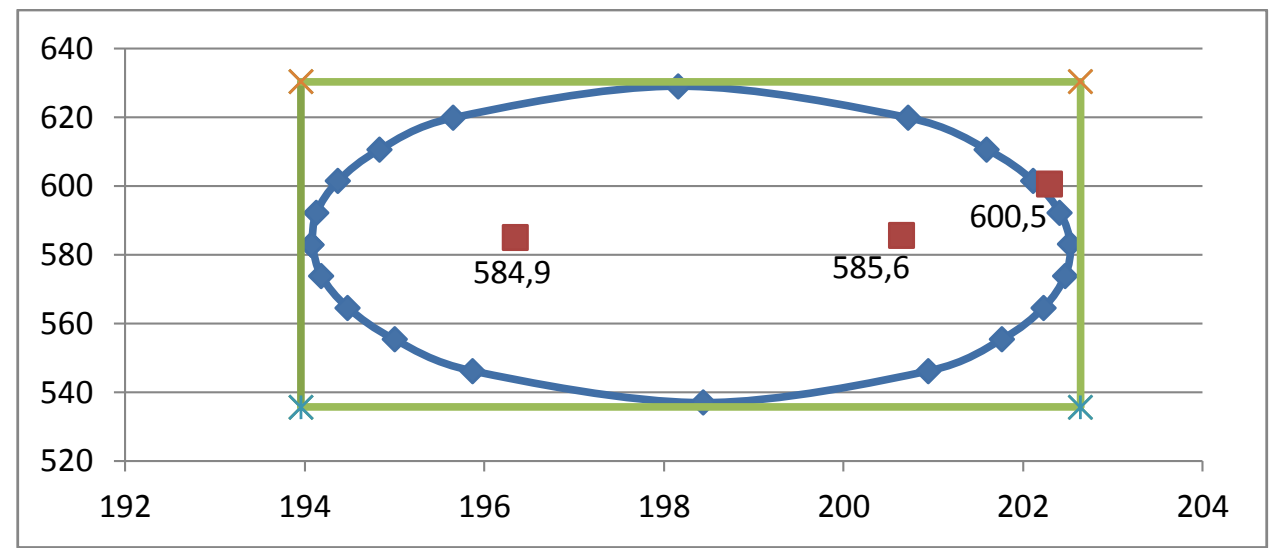

Рисунок 1 - Контрольная карта Хоттелинга

На рис.1 прямоугольником обозначена доверительная область для независимых критериев, эллипс соответствует многомерному контролю. Точками отмечены средние значения выборок. Важно отметить, что значение статистики Хоттелинга для третьей выборки оказывается выше критического, и точка лежит вне эллипса, однако внутри прямоугольника. Таким образом, учет взаимного влияния технологических параметров друг на друга позволяет более точно оценивать статистическую управляемость процесса производства.

\section{Список литературы}

1. Кузнецов Л.А., Погодаев А.К., Корнеев А.М. Статистические модели в задачах оптимизации сквозной технологии производства автолистовой стали Известия вузов. Черная металлургия, 1990, №3, с.34-36.

2. ГОСТ Р 50 779.42-99. Статистические методы. Контрольные карты Шухарта. М.: Изд-во стандартов, 1999. 32с.

3. Шторм Р. Теория вероятностей. Математическая статистика. Статистический контроль качества: пер. с не. под ред. Н.С. Райбмана. М.: Мир, 1970. 368 с.

4. Корнеев, А.М. Методы идентификации сквозной технологии производства металлопродукции [Текст]: монография / A.M. Корнеев; Липецкий государственный педагогический университет. - Липецк: ЛГПУ, 2009. - 286 с.

5. Кузнецов Л.А., Корнеев А.М. Автоматизированная система выбора оптимальной технологии производства проката. - Известия вузов. Черная металлургия, 1994, №5, c. $45-48$. 